\title{
Multispectral Imaging
}

National Cancer Institute

\section{Source}

National Cancer Institute. Multispectral Imaging. NCI Thesaurus. Code C116495.

An imaging technique that captures data at multiple frequencies across the

electromagnetic spectrum, including those from visible and non-visible wavelengths. 